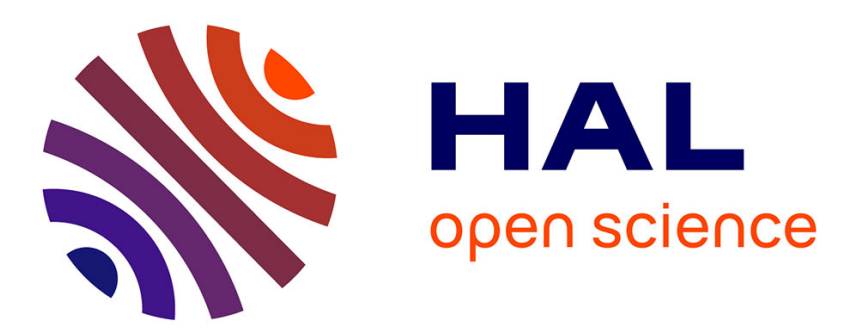

\title{
Bayesian Networks Implementation of the Dempster Shafer Theory to Model Reliability Uncertainty
}

\author{
Christophe Simon, Philippe Weber
}

\section{To cite this version:}

Christophe Simon, Philippe Weber. Bayesian Networks Implementation of the Dempster Shafer Theory to Model Reliability Uncertainty. Workshop on Bayesian Networks in Dependability (BND2006) in the First International Conference on Availiability, Reliability and Security, ARES 2006, Vienna, Austria, April 20-22, 2006., 2006, Vienna, Austria. pp.788-793. hal-00023393

\section{HAL Id: hal-00023393 https://hal.science/hal-00023393}

Submitted on 26 Apr 2006

HAL is a multi-disciplinary open access archive for the deposit and dissemination of scientific research documents, whether they are published or not. The documents may come from teaching and research institutions in France or abroad, or from public or private research centers.
L'archive ouverte pluridisciplinaire HAL, est destinée au dépôt et à la diffusion de documents scientifiques de niveau recherche, publiés ou non, émanant des établissements d'enseignement et de recherche français ou étrangers, des laboratoires publics ou privés. 


\title{
Bayesian Networks Implementation of the Dempster Shafer Theory to Model Reliability Uncertainty
}

\author{
Christophe SIMON, Philippe WEBER \\ CRAN UMR 7039, CNRS-UHP-INPL \\ ESSTIN, 2 Rue Jean Lamour, 54519 Vandoeuvre, France \\ Phone: (++33) 383-685-134, email: christophe.simon@esstin.uhp-nancy.fr
}

\begin{abstract}
In many reliability studies based on data, reliability engineers face incompleteness and incoherency problems in the data. Probabilistic tools badly handle these kinds of problems thus, it is better to use formalism from the evidence theory. From our knowledge, there is a lack of industrial tools that implement this theory. In this paper, the implementation of the Dempster Shafer theory in a Bayesian Network tool is proposed in order to compute system reliability and manage epistemic uncertainty propagation. The basic concepts used are presented and some numerical experiments are made to show how uncertainty is propagated.
\end{abstract}

\section{Introduction}

Bayesian Networks bring a solution to estimate system performances by concentrating in a compact structure the states of component functioning and provide a causal or diagnostic analysis of the system [3]. Works on system safety and Bayesian Networks were developed by Boudali and Dugan [2] and Bobbio, et al., [1] who explained how fault trees can be implemented by using Bayesian Networks. Moreover investigations are made in dynamic reliability analyses, the paper [23] describes the reliability modelling with a dynamic Bayesian Network that simulates a stochastic process with exogenous constraints. The dynamic model proposed by Weber, et al. [22] is built from the analysis of a system functioning and malfunctioning. This model allows computing of state probability distributions by taking into account both the age of components and maintenance operations.

From an industrial point of view, due to the complexity of a system, it is classically accepted that observations made on the system are partially realised. For instance, the observations recorded in data can be done after inspection or maintenance action therefore the complete knowledge is not available at any time. During the use of these data with uncensored measures, we can point out an epistemic uncertainty on the state of the component and its influence on the state of the system. In the probability framework, incomplete data should be censored or completed according to the principle of minimal commitment. It corresponds to the maximum entropy principle. The corresponding probability masses are equally distributed on each possible state. In the evidence framework, the belief mass associated to the incomplete data, that is to say uncertainty on the state, is allocated to the uncertain state modality according to the minimal commitment principle. Thanks to the evidence theory formalism, the principle of minimal commitment formalizes the idea that we should never give more support than justified to any subset of the frame of discernment [18]. Consequently, the probability framework imposes a random view of uncertainty that is debatable. Therefore, the framework of the evidence theory and more precisely the Dempster-Shafer theory seems more appropriate to express and process the epistemic uncertainty.

Bayesian Networks are powerful modelling tools when the problem is handled under a frequentist or subjectivist point of view [19]. In addition, Valuable Networks [16, 7] are powerful tools that are well adapted when the modelling problem is handled under the knowledge point of view. Shenoy, et al [5] have shown the advantages and drawbacks of these tools. Moreover, they have proved their equivalence under some conditions.

In this article, we propose a particular integration of the Dempster-Shafer theory in the Bayesian Network tool in order to handle the epistemic uncertainty problem. Moreover, we take advantage of the power of the Bayesian Network tools to model system reliability. Thus, the second section of the paper is dedicated to the basics of the evidence theory. Moreover, the paper shows how exact inference algorithms used by the software simulating Bayesian Networks provide a support to the simulation of the evidence theory applied to the reliability evaluation. The third section of 
the paper deals with an application to the reliability analysis of the bridge system and how the epistemic uncertainty is propagated through the Bayesian Network.

\section{The basics of the evidence theory}

The evidence theory sometimes called belief functions has been initiated by Dempster with his works on milestones superior and inferior bounds of a family of probability distributions [8] then reinforced by Shafer [15]. Different processing models of imperfect information have thus appeared: upper and lower model [21]; Dempster-Shafer Theory and Hint Model of Kholas and Monney [12]; transferable Belief Model [18].

The model of Dempster-Shafer is a generalized Bayesian model. The idea of this model is to allocate a number between 0 and 1 to indicate a degree of belief on a proposal as in the probability framework. Nevertheless, it is not considered a probability but a belief mass. The distribution of masses is called basic belief assignment. As in the probability framework, the following allocation function is found: $m: 2^{\Omega} \rightarrow[0,1]$ such that:

$$
m(\emptyset)=0 ; \sum_{A \in \Omega} m(A)=1
$$

where $\Omega$ is the frame of discernment. This frame is the set of disjoint component states or reasoning modalities. The basic belief assignment concerns the possibility to assign masses to every $2^{\Omega}$ combinations and then expressing epistemic uncertainty.

\subsection{Basic belief assignment}

The frame of discernment used in reliability study is generally described by the following equations:

$$
\Omega=\{U p, \text { Down }\}, \quad m: 2^{\Omega} \rightarrow[0,1]
$$

And

$$
2^{\Omega}=\left\{\begin{array}{cc}
m(\emptyset)=0 ; & m(\{U p\}) \\
m(\{\text { Down }\}) ; & m(\{U p \text { or Down }\})
\end{array}\right.
$$

Under the following constraint: $\sum_{A \in \Omega} m(A)=1$

The basic belief assignment is possible on the $\{U p$ or Down $\}$ modality where $\{U p\}$ and $\{$ Down $\}$ are the two disjoint states of a component. This basic belief assignment can be considered as a priori probability for root nodes in evidence networks. It is called a priori belief mass.

The frame previously defined is the one commonly used in reliability studies. Besides, it corresponds to assumptions made in fault tree analyses. Currently, reliability engineers extend this framework to multistate components. In this case, the number of combinations in the allocation function $m$ increases and consequently the number of epistemic modalities also increases. This is a well known practice problem in evidence theory. Nevertheless, in the case of reliability studies based on databases, basic belief assignment are computed directly from data and this computing mitigate engineers' efforts.

\section{$2.2 \quad$ Evidence inference}

In the literature, the inference algorithm based on principles stated by Pearl [14] is recommended to propagate calculations in an evidence network. Recent algorithms developed for the inference in Bayesian Networks as junction trees by Jensen [11] provide a more efficient solution to propagate calculations in acyclic oriented graphs such as evidence networks. We propose to use this exact inference algorithm to simulate the behaviour of an evidence network in the Bayesian Network software (Bayesialab(c).

To compute the system reliability, we should model conjunctive and disjunctive operators and integrate them in Bayesian Networks tool to replace conditional probability tables. These operators take into account the particular $\{U p$ or Down $\}$ modality into the Bayesian inference. The truth tables have been given by Guth in [9] and transcribed in network nodes (see figures 1 and 2). As one can see in these tables, the main difficulty is to define the relation between $\{U p$ or Down $\}$ input modality and all output modalities. For an AND gate between two inputs (figure 1) if one input is in state $\{U p\}$ then the output is in the same state as the second input. If one input is in state $\{$ Down $\}$ then there is no ambiguity for the output which is obviously in state $\{D o w n\}$. If one input is in uncertain state $\{U p$ or Down $\}$ then two cases are encountered. Either the second input is in state $\{U p\}$ or $\{U p$ or Down $\}$ then the output is in state $\{U p$ or Down $\}$. If the second input is in state $\{$ Down $\}$ then it is sure that the output is in state $\{$ Down $\}$.

Tables 1 and 2 are called conditional belief tables. They are introduced in a Evidence Network implemented by the industrial tool Bayesialab as shown on figure 6. Nodes contain the conjunctive or disjunctive operator chosen according to the reliability problem modelled.

If the reliability analysis is extended to multistate components but restricted to series and/or parallel connections between subsystems, some efforts should be made on conditional belief tables. For example, if a three-state reasoning is introduced for a subsystem composed of 2 components and the output is also based on three states, the number of parameters for the conditional belief tables will increase to $7^{3}$. Practically, conditional belief tables are equal to the 


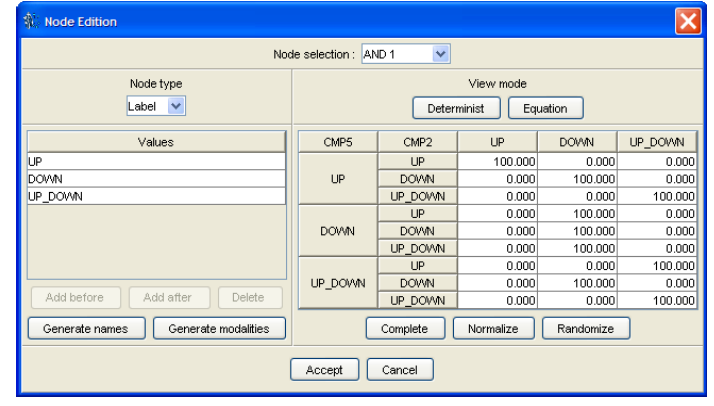

Figure 1. Conditional belief Table for AND gate.

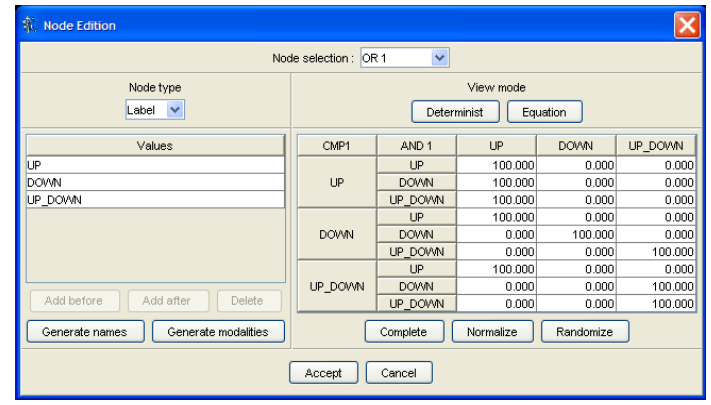

Figure 2. Conditional belief Table for OR gate.

truth tables of an AND or OR Gates. Thus, only the relation between the basic disjoint modalities of the inputs (not the epistemic modalities) should be defined. The relations between all other modalities were deduced directly from De Morgan laws.

\subsection{Plausibility and belief functions}

Once the reliability engineer has defined the basic belief assignment for each component and the network has computed the assignment for each modality of the system state, we expect information about the system failure probability. In the Dempster Shafer Theory, two main functions help us obtain some information about this probability, plausibility and belief functions.

The plausibility function characterizes the degree in which a proposal $A$ is plausible based on available evidence $B$ expressed by each basic belief mass that contributes to the realization of $A$. This function is defined as $p l s: 2^{\Omega} \rightarrow[0,1]$ computed by the following equation:

$$
p l s(A)=\sum_{B \mid A \cap B \neq \emptyset} m(B)
$$

A belief function exists and characterizes the degree in which a proposal $A$ is believable based on available evidence expressed by each basic belief mass assigned on

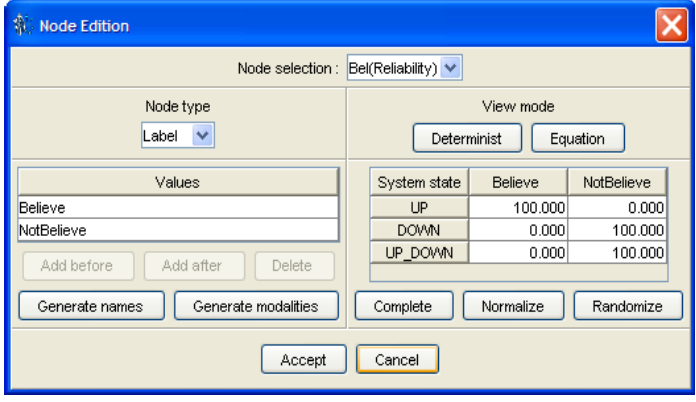

Figure 3. Table of bel (Reliability).

the frame of discernment. This function is defined as bel : $2^{\Omega} \rightarrow[0,1]$ computed by the following equation:

$$
\operatorname{bel}(A)=\sum_{B \mid B \subseteq A} m(B)
$$

Using these calculus rules, we obtain an interesting characteristic that is the bounding of the probability by the measures of belief and plausibility [13].

$$
\operatorname{bel}(A) \leq \operatorname{Pr}(A) \leq p l s(A)
$$

This property is well known and Shafer has already define it in his works in 1976 [15], and many authors have used this relation between interval $[b e l, p l s]$ and basic belief assignment ([9], [4], [10]).

To compute belief and plausibility functions within a Bayesian Network tool, two different nodes (bel, pls) should be defined according to the conditional belief tables shown on figures 3 and 4 . These tables transcribed equations 1 and 2. As Bayesian Networks respect the additive constraint $\left(\sum_{a \in \Omega} \operatorname{Pr}(a)=1\right)$, belief and plausibility functions should be computed in two separate nodes as shown on the top of figure 6 . This is the only solution to release this constraint. Nevertheless, the additive constraint should be respected inside each node. Thus, we need to introduce a Notbelieve (resp. Implausibility) modality in the believe node (resp. plausibility node).

One can note that these tables and the network structure (figure 6) can also be used to compute plausibility and belief functions on each component or node in order to have information on probability boxes that bound the real value of the failure probability for each subsystem. 


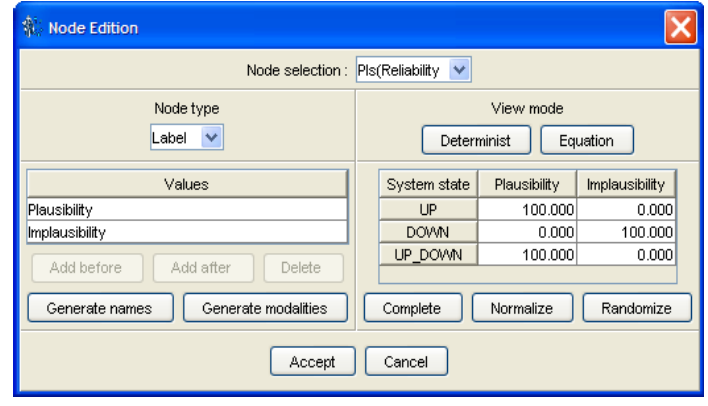

Figure 4. Table of $p l s$ (Reliability).

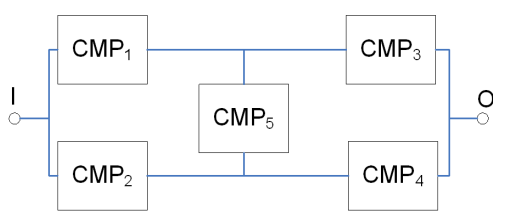

Figure 5. Reliability diagram

\section{Application to reliability}

To argue the interest of the approach, we used the bridge system previously defined by Torres-Toledano in [20]. This system is a simple study case, however it allows to understand all the problematic of the reliability modelling in its general frame. Its Bayesian Network model to compute reliability has been given by Torres-Toledano and Sucar [20] and its reliability diagram is given on figure 5 .

\subsection{A case study}

The case study is composed of five components $C_{1}$ to $C_{5}$. Each component can be in one of the two disjoint states $\{U p\}$ and $\{$ Down $\}$. The elementary events $E_{i}$ that is to say the failures driving the component from state $\{U p\}$ to state $\{$ Down $\}$ are independent. The system is homogeneous and no repairs are considered. These assumptions are usual in reliability studies and do not reduce the generality of the case study.

According to these assumptions, let us defined numerical values for failure rates of each component $\left(\lambda_{1}=\lambda_{2}=\right.$ $\left.\lambda_{5}=10^{-3} h^{-1} ; \lambda_{3}=\lambda_{4}=2.10^{-3} h^{-1}\right)$.

The time of mission of the system is $T_{F}=200 h$, then $R_{1}\left(T_{F}\right)=R_{2}\left(T_{F}\right)=R_{5}\left(T_{F}\right)=0,81873$ and $R_{3}\left(T_{F}\right)=R_{4}\left(T_{F}\right)=0,67032$.

\subsection{The total probability theorem}

To compute the exact value of the reliability of the system, a formal way through the total probability theorem is

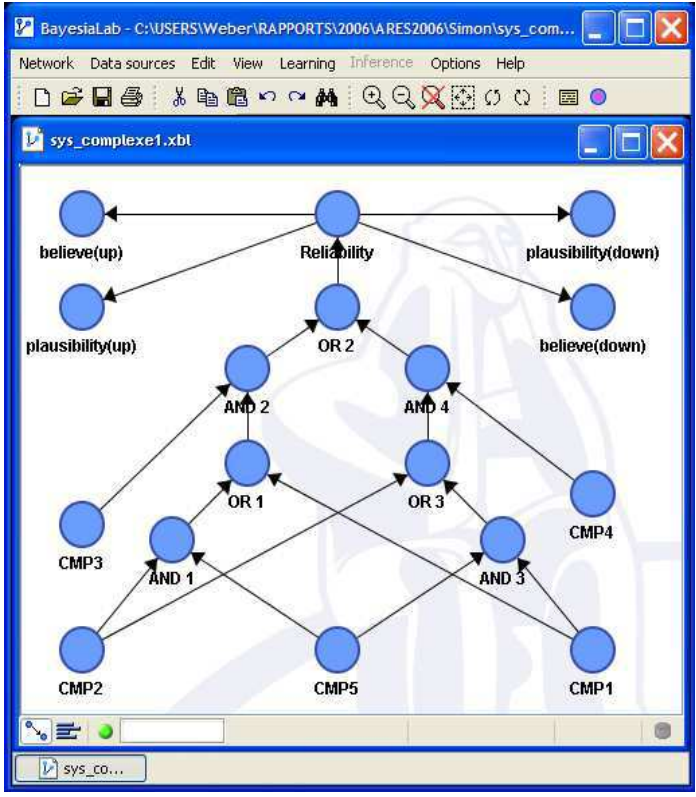

Figure 6. Evidence Network.

investigated. In this method, two structures should be considered according to the state of $C_{5}$. The total probability theorem helps to write the equation of the system reliability $R_{s}(t)$ :

$$
\begin{aligned}
& R_{s}(t)=P\left(S \text { is }\{U p\} \mid C_{5} \text { is }\{U p\}, t\right) \cdot R_{5}(t) \\
& +P\left(S \text { is }\{U p\} \mid C_{5} \text { is }\{\text { Down }\}, t\right) \cdot\left(1-R_{5}(t)\right)
\end{aligned}
$$

Where $R_{i}(t)$ is the reliability of the component $C_{i}$ according to the time $t$. By relation 4 , the value of the reliability $R_{S}\left(T_{F}\right)=0,85013$ is obtained.

\subsection{The evidence network model}

Let us consider the Bayesian Network tool Bayesialab@(http://www.bayesia.com) implementing the Dempster Shafer Theory and lets it apply to the case study. The structure of the evidence network model is presented on figure 6. For evidence networks, a priori belief masses have been defined according to the reliability of each component. Figure 7 shows how a priori belief masses are assigned for component $C_{1}$.

\subsection{A case with no epistemic uncertainty}

According to the evidence network shown on figure 6, the reliability of the system is evaluated thanks to the inference algorithm. The result is exactly equal to the one com- 


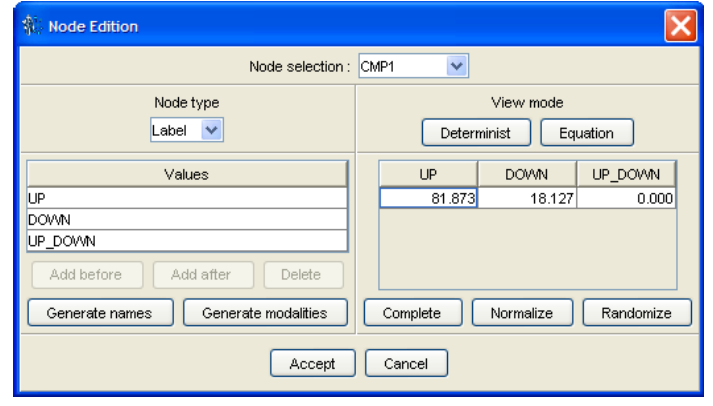

Figure 7. a priori belief mass assignment on $C_{1}$.

\begin{tabular}{|l|l|l|l|}
\hline & Up & Down & Up or Down \\
\hline$R_{s}\left(T_{F}\right)$ & 0,85013 & 0,14987 & 0 \\
\hline
\end{tabular}

Table 1. System reliability by inference without uncertainty

puted by the total probability theorem. It confirms the exactness of evidence networks. If no epistemic uncertainty is introduced in component states $(m(\{U p$ or Down $\})=0)$, then the reliability value previously computed is confirmed.

Obviously, the Dempster Shafer Theory offers a general Bayesian framework entirely compatible with the probabilistic one. Results without uncertainty can be observed on figure 8 and the following condition is obtained: $\operatorname{bel}\left(R_{s}\left(T_{F}\right)\right.$ is $\left.\{U p\}\right)=\operatorname{Pr}\left(R_{s}\left(T_{F}\right)\right.$ is $\left.\{U p\}\right)=$ $p l s\left(R_{s}\left(T_{F}\right)\right.$ is $\left.\{U p\}\right)=0,85013$.

\subsection{A case with an epistemic uncertainty}

Let us consider the case of an epistemic uncertainty introduced on the state of one component. The propagation of this uncertainty is observed in the Bayesian Network tool (see figures 9 and 10). According to the importance of the component in the system and the quantity of uncertainty, the

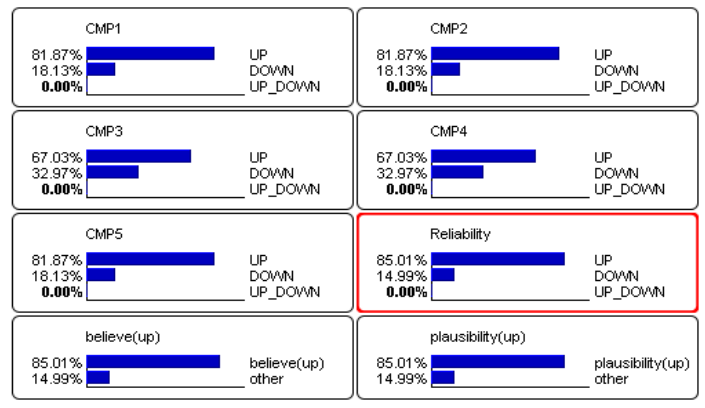

Figure 8. Inference results $\left(m\left(C_{1}=0\right)\right)$.

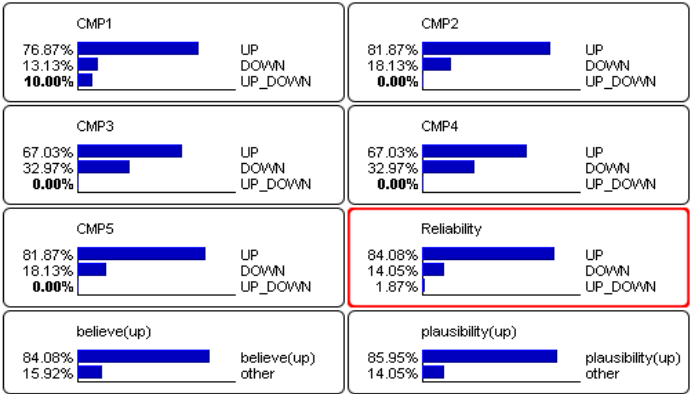

Figure 9. Inference results ( $\left.m\left(C_{1}=0,1\right)\right)$.

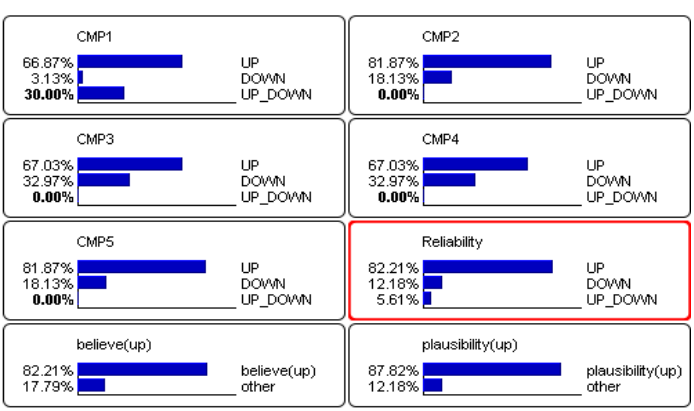

Figure 10. Inference results $\left(m\left(C_{1}=0,3\right)\right)$.

epistemic uncertainty on the system reliability can vary importantly. For example, if a mass of epistemic uncertainty $m\left(C_{1}\right.$ is $\{U p$ or Down $\left.\}\right)=0,1$ is introduced in component $C_{1}$ then:

$$
\begin{aligned}
& \operatorname{bel}\left(R_{s}\left(T_{F}\right) \text { is }\{U p\}\right)=0,8408 \\
& \operatorname{Pr}\left(R_{s}\left(T_{F}\right) \text { is }\{U p\}\right)=0,85013 \\
& \text { pls }\left(R_{s}\left(T_{F}\right) \text { is }\{U p\}\right)=0,8595
\end{aligned}
$$

This calculus leads to $m\left(R_{s}\left(T_{F}\right)\right.$ is $\{U p$ or Down $\left.\}\right)=$ 0,0187 (figure 9). If the uncertainty on $C_{1}$ grows up to 0,3 $m\left(R_{s}\left(T_{F}\right)\right.$ is $\{U p$ or Down $\}$ ) goes to 0,0561 (figure 10) and the following values are obtained:

$$
\begin{aligned}
& \operatorname{bel}\left(R_{s}\left(T_{F}\right) \text { is }\{U p\}\right)=0,8221 \\
& \operatorname{Pr}\left(R_{s}\left(T_{F}\right) \text { is }\{U p\}\right)=0,85013 \\
& \text { pls }\left(R_{s}\left(T_{F}\right) \text { is }\{U p\}\right)=0,8782
\end{aligned}
$$

If the basic belief assignment on the system state expresses an epistemic uncertainty then bel and $p l s$ measures are not equal and bound the system reliability.

In the framework of the evidence theory, we recognize a two-level mental model: the creedal level where beliefs are held and represented by belief functions, and the pignistic level where decisions are made [17]. Many transformation functions can be found: the pignistic transformation defined by smets [17] or the plausibility transformation [6]. In this paper, we have chosen to work on the credal level and manipulate the basic belief assignments and their propagation 
through Bayesian Networks inference. The goal is to help the reliability engineer to handle the epistemic uncertainty of the system state according to the epistemic uncertainty of the state of the components. The belief measure informs the reliability engineer about the worst value of the reliability of the system. By running diagnostic or intercausal modes of Bayesian Networks inference, the reliability engineers can identify the components which introduce the epistemic uncertainty on the state of the system. Then, he can plan all needed inspection to reduce this uncertainty.

\section{Conclusion}

In this paper, the powerful representation and the exactness of Bayesian Networks in reliability studies are shown. In some context such as incomplete data in databases or incoherency between data and reliability models, we can use uncensored data with the Dempster Shafer Theory to take the epistemic uncertainty into account. The paper has shown how basic concepts of the Dempster Shafer Theory can be implemented in Bayesian Networks tool to manage this kind of uncertainty and to extract the most of information from available data.

\section{References}

[1] A. Bobbio, L. Portinale, M. Minichino, and E. Ciancamerla. Improving the analysis of dependable systems by mapping fault trees into bayesian networks. Reliability Engineering and System Safety, 71(3):249-260, March 2001.

[2] H. Boudali and J. Dugan. A discrete-time bayesian network reliability modeling and analysis framework. Reliability Engineering and System Safety, 87:337-349, 2005.

[3] M. Bouissou and O. Pourret. A bayesian belief network based method for performance evaluation and troubleshooting of multistate systems. International Journal of Reliability, Quality, and Safety Engineering, 10(4):407-416, 2003.

[4] Y. Cheng. Uncertainty in fault tree analysis. Tamkang Journal of Science and Engineering, 3(1):23-29, 2000.

[5] B. Cobb and P. Shenoy. A comparison of bayesian and belief function reasoning. Technical Report 292, University of Kansas, School of Business, September 2002.

[6] B. Cobb and P. Shenoy. On the plausibility transformation method for translating belief function models to probability models. International Journal of Approximate Reasoning, 2005.
[7] R. Demirer and P. Shenoy. Sequential valuation networks: A new graphical technique for asymmetric decision problem. Lecture Notes in Computer Science, pages 252-265, 2001.

[8] A. Dempster. Upper and lower probabilities induced by a multivalued mapping. Annals of mathematical statistics, 38:325-339, 1967.

[9] M. Guth. A probability foundation for vagueness and imprecision in fault tree analysis. IEEE Transactions on Reliability, 40(5):563-570, 1991.

[10] J. Hall and J. Lawry. Imprecise probabilities of engineering system failure from random and fuzzy set reliability analysis. In ISIPTA'01, 26-29 june 2001.

[11] F. Jensen. An Introduction to Bayesian Networks. London, 1996.

[12] J. Kholas and P. Monney. A mathematical Theory of Hints: An approach to Dempster-Shafer Theory of evidence, volume 425. Springer Verlag, 1995.

[13] G. Klir and B. Yuan. Fuzzy Sets and Fuzzy Logic. Theory and Applications. NJ:Prentice Hall, 1995.

[14] J. Pearl. Probabilistic Reasoning in intelligent Systems: Networks of Plausible Inference. 1988.

[15] G. Shafer. A mathematical theory of evidence. Princeton University Press, 1976.

[16] P. Shenoy. Valuation based systems: a framework for managing uncertainty in expert systems, pages 83-104. Wiley, New-York, 1992.

[17] P. Smets. Decision making in the tbm: the necessity of the pignistic transformation. International Journal of Approximate Reasoning, 38:133-147, 2005.

[18] P. Smets and R. Kennes. The transferable belief model. Artificial Intelligence, 66:191-243, 1994.

[19] D. Spiegelhalter, A. Dawid, S. Lauritzen, and R. Cowell. Bayesian analysis in expert systems. Statistical Science, 8:219-283, 1993.

[20] J. Torres-Toledano and L. Sucar. Bayesian networks for reliability of complex systems. In H. Coelho, editor, Progress in Artificial Intelligence, IBERAMIA98, pages 195-206. Springer-Verlag, 2004.

[21] P. Walley and S. Moral. Upper probabilities based on the likelihood function. Journal of Royal Society B, 61(4):831847, 1999.

[22] P. Weber and L. Jouffe. Complex system reliability modelling with dynamic object oriented bayesian networks (doobn). Reliability Engineering and System Safety, 91(2):149-162, February 2006.

[23] P. Weber, P. Munteanu, and L. Jouffe. Dynamic bayesian networks modelling the dependability of systems with degradations and exogenous constraints. In INCOM'04, April 5-7 2004. 